\title{
AVALIAÇÃO FLUIDODINÂMICA DA SECAGEM DE SUSPENSÃO DILUIDA EM LEITO FLUIDIZADO UTILIZANDO ANÁLISE ESPECTRAL GAUSSIANA
}

\author{
F.T.S. SCHAFFKA ${ }^{1}$, C.A.M. SILVA ${ }^{2}$, F.C. JANZEN ${ }^{3}$, M.R. PARISE ${ }^{4}$ \\ ${ }^{1}$ Universidade Tecnológica Federal do Paraná- Campus Ponta Grossa, Departamento \\ de Engenharia Mecânica \\ ${ }^{2}$ Universidade Estadual de Campinas, Faculdade de Engenharia Química \\ ${ }^{3}$ Universidade Tecnológica Federal do Paraná- Campus Ponta Grossa, Departamento de \\ Engenharia Eletrônica \\ ${ }^{4}$ Universidade Tecnológica Federal do Paraná- Campus Ponta Grossa, Departamento \\ de Engenharia Química \\ *email:parise@utfpr.edu.br
}

\begin{abstract}
RESUMO
O presente trabalho consiste em avaliar as transições de regime de fluidização durante o processo de secagem de suspensão diluída utilizando a análise espectral Gaussiana, que se baseia na medição das flutuações de pressão no leito. Os ensaios experimentais foram realizados em escala laboratorial composto por uma coluna de acrílico de $0,11 \mathrm{~m}$ de diâmetro interno e 1,0 m de altura. $\mathrm{O}$ equipamento experimental foi instrumentado com transdutores de pressão de resposta rápida, termopares $\mathrm{e}$ termohigrômetros acoplados a um sistema de aquisição e processamento de sinais, composto por uma placa de aquisição de dados, um microcomputador e pelo software LabView $10.0^{\mathrm{TM}}$. Esferas de alumínio com diâmetro médio de partícula de $1,55 \mathrm{~mm}$ foram usadas como material inerte, e suspensão de carbonato de cálcio com concentração de $9 \%$, em massa, foi utilizada como material pastoso pulverizado sobre o leito. A vazão de atomização da suspensão foi de 12 e $15 \mathrm{~mL} / \mathrm{min}$. Os resultados mostraram que a transição dos regimes de fluidização durante o processo de secagem de suspensão aquosa pode ser monitorada com a técnica da análise espectral Gaussiana.
\end{abstract}

\section{INTRODUÇÃO}

As indústrias de alimentos, químicas e farmacêuticas têm usado extensivamente leitos fluidizados em diversos tipos de processamento, devido ao contato efetivo entre as partículas sólidas e um gás ou líquido, permitindo um processo com altas taxas de transferência de calor e de massa.
Nos processos que utilizam leito fluidizado gás-sólido é fundamental manter a estabilidade do estado de fluidização, de modo a obter uma boa mistura das fases gasosa e sólida e, consequentemente, alto desempenho nos processos.

Porém, temperaturas elevadas, alto conteúdo de umidade de partículas, e até mesmo, em processos em que uma solução 
tem de ser atomizada diretamente em um leito fluidizado, como é o caso da secagem de suspensão diluída, revestimento das partículas e granulação, pode ser difícil garantir a qualidade de fluidização, devido ao alto conteúdo de umidade no leito tender à aglomeração das partículas e acelerar a condição de defluidização (MARONGA, 1998).

Vários métodos têm sido estudados para quantificar os regimes de fluidização, e assim avaliar a qualidade do movimento das partículas (BOOK et al., 2011; SILVA et al., 2014; SHABANIAN al., 2015, SILVA, 2015). Dentre esses estão inseridas as análises no domínio do tempo, espectral, wavelet e de caos. PARISE (2007) e PARISE et al. (2008) desenvolveram a análise espectral Gaussiana para identificar a região de defluidização do leito, utilizando medidas de flutuação de pressão no plenum. Com essa técnica é possível avaliar o momento em que o leito está tendendo à defluidização através de alterações significativas dos valores da freqüência central. SILVA et al. (2009) e SILVA et al. (2011) aplicaram essa técnica no processo de recobrimento de partículas de celulose microcristalina em leito fluidizado atomizando água e suspensão polimérica, respectivamente. Com a técnica empregada, a defluidização do leito foi facilmente detectada em ambos os estudos.

Neste sentido, este trabalho tem como objetivo a realização de um estudo do comportamento fluidodinâmico no processo de secagem de suspensão diluída de carbonato de cálcio com concentração em massa de $9 \%$ em um leito composto por partículas inertes de esferas de alumínio $\left(\mathrm{d}_{\mathrm{p}}=1,55 \mathrm{~mm}\right)$, utilizando análise espectral Gaussiana, que é uma técnica utilizada em medidas de flutuação de pressão no leito.

\section{MATERIAIS E MÉTODOS}

Os experimentos foram realizados em um secador de leito fluidizado em escala de laboratório como mostrado na Figura 1. O sistema é composto por uma coluna de acrílico de $0,11 \mathrm{~m}$ de diâmetro interno e 1,0 m de altura. $\mathrm{O}$ ar utilizado para a fluidização das partículas foi fornecido por um soprador radial da marca Elan ${ }^{\circledR}$, modelo CRE04, aclopado a um motor de $4 \mathrm{cv}$. Este soprador está conectado ao leito através de uma tubulação de aço com $0,038 \mathrm{~m}$ de diâmetro interno. A rotação do motor do soprador foi regulada com auxílio de um inversor de frequência da marca $W E G \AA$, modelo CFW080160T2024POA1Z. A vazão mássica do ar destinado ao leito de partículas foi determinada mediante uma placa de orifício (construída a partir da Norma NBR-ISO 5167-1, ABNT, 1994). Para isso, a pressão manométrica na linha e a queda de pressão através da placa de orifício foram obtidas utilizando transdutores de pressão (Cole Parmer ${ }^{\circledR}$, modelo 98073-14, de 0 a $12442 \mathrm{~Pa}$, e do modelo 68071-14, de 0 a $6227 \mathrm{~Pa}$, respectivamente). $\mathrm{O}$ ar fluidizante era aquecido através de resistências elétricas e um regulador tipo PID $\left(\mathrm{NOVUS}^{\circledR}\right.$, modelo N1100) foi utilizado para controlar a temperatura do ar no plenum. Os sinais de pressão provenientes do plenum foram obtidos utilizando um transdutor diferencial de pressão (Cole Parmer ${ }^{\circledR}$, modelo 68071-14, com faixa de 0 a $6227 \mathrm{~Pa}$ ). Todos os transdutores de pressão utilizados foram acoplados a um sistema de aquisição de dados (National Instruments ${ }^{\mathrm{TM}}$, modelo NI USB 6211). Foram utilizados dois sensores de umidade relativa do ar e temperatura de bulbo seco (Novus, modelos: RHT-XS e N322 RHT). Para as medidas de temperatura na coluna de fluidização, foram utilizados termopares tipo $\mathrm{T}$, conectados a um sistema de aquisição de dados (National Instruments $^{\mathrm{TM}}$, modelo NI USB 9213). Os sinais de pressão, umidade e temperatura foram visualizados e processados a partir de 
um microcomputador com software LabView $^{\text {TM }}$ versão 10.0. Foram coletados 2048 pontos de pressão no plenum a uma taxa de amostragem de $400 \mathrm{~Hz}$. Um bico atomizador do tipo duplo fluido (Spraying Systems ${ }^{\circledR}$, SU11-SS) foi utilizado para atomizar a suspensão diluída provinda de uma bomba peristáltica (Vallair $^{\circledR}$, modelo SVP4H2B1SZAA). O ar de atomização foi fornecido por compressor do tipo pistão $\left(\right.$ Schulz $^{\circledR}$, modelo 821410, $\left.2 \mathrm{cv}\right)$ o qual passava por um regulador de pressão (Fluir ${ }^{\mathbb{R}}$, modelo AW200002U). Os pós secos eram coletados por um ciclone Swift conectado a um filtro de mangas tipo jato pulsante $\left(\right.$ Multiman $\left.^{\circledR}\right)$.

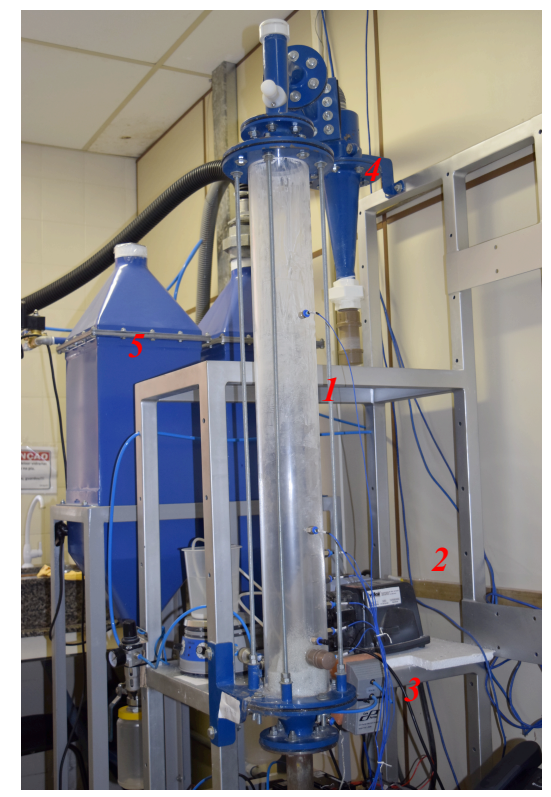

Figura 1 - Fotografia do sistema experimental. 1: coluna de fluidização; 2: bomba peristáltica; 3: transdutor de pressão; 4: ciclone; 5: filtro de mangas.

O material inerte utilizado nos experimentos foi esferas de alumínio e a suspensão diluída pastoso é composta por carbonato de cálcio e água destilada. As características físicas da esfera de alumínio são ilustradas na Tabela 1 .
Tabela 1 - Características físicas do material inerte.

\begin{tabular}{ccc}
\hline Material & $\begin{array}{c}\text { Diâmetro médio } \\
\text { das partículas } \\
(\mathrm{mm})\end{array}$ & $\begin{array}{c}\text { Densidade } \\
\text { aparente } \\
\left(\mathrm{kg} / \mathrm{m}^{3}\right)\end{array}$ \\
\hline $\begin{array}{c}\text { Esferas de } \\
\text { alumínio }\end{array}$ & $1,55(+1,4-1,7)$ & 2692,60 \\
\hline
\end{tabular}

Em todos os ensaios experimentais, a altura de leito fixo manteve-se $0,10 \mathrm{~m}$ e a massa de material inerte foi de $1,60 \mathrm{~kg}$. A Tabela 2 mostra as condições operacionais utilizadas.

Tabela 2 - Condições experimentais dos experimentos.

\begin{tabular}{lccc}
\hline Ensaio & $\mathrm{u}_{\mathrm{o}} / \mathrm{u}_{\mathrm{mf}}$ & $\mathrm{C}_{\text {suspensão }}(\%)$ & $\begin{array}{c}\mathrm{Q}_{\text {suspensão }} \\
(\mathrm{mL} / \mathrm{min})\end{array}$ \\
\hline 1 & 1,4 & 9,0 & 12,0 \\
2 & 1,4 & 9,0 & 15,0 \\
\hline
\end{tabular}

\subsection{Análise espectral Gaussiana}

A metodologia de análise espectral Gaussiana foi desenvolvida por Parise (2007) e será utilizada para identificar a transição de regime fluidodinâmico durante processo de secagem de suspensão diluída em leito fluidizado. Para essa finalidade, inicialmente, são coletados 2048 pontos de pressão manométrica no plenum a uma frequência de amostragem de $400 \mathrm{~Hz}$ (este valor de frequencia tem sido usado por vários pesquisadores, como por exemplo, BRIENS et al, 2003; BAI et. al, 2005, PARISE, 2008; PARISE et. al. 2009, SILVA et. al. 2011 e SILVA 2015). Após esse procedimento, esses sinais de pressão são filtrados utilizando filtro digital (IRR- resposta de impulso infinito - do tipo passa baixa, com topologia Butterworth, frequênica de corte de $20 \mathrm{~Hz}$ ) e realiza-se a centralização na média (mean centring). A Transformada Rápida de Fourier (FFT) é aplicada neste sinal de pressão centralizada, produzindo um espectro de pressão. Em seguida, é realizado um ajuste deste sinal na curva dada pela Equação 1: 
$G\left(f_{k}\right)=A e^{\frac{-\left(f_{k}-f_{m}\right)}{2 \sigma^{2}}}$

Aplicando o logaritmo natural na Equação 1 e utilizando o método dos mínimos quadrados, dado pela Equação 2, a frequência central, $f_{c}$, pode ser determinada.

$$
e_{k}=\sum_{k=0}^{N-1}\left\{\ln \left[A_{e}\left(f_{k}\right)\right]-\ln \left[G\left(f_{k}\right)\right]\right\}^{2}
$$

O valor da frequência central é o parâmetro utilizado para o estudo da transição de regimes de fluidização do presente trabalho.

\section{RESULTADOS E DISCUSSÃO}

As Figuras 3 e 4 mostram o perfil da frequência central ao longo do tempo de secagem de suspensão diluída de carbonato de cálcio para a vazão de atomização de 12 e $15 \mathrm{~mL} / \mathrm{min}$, respectivamente.

$\mathrm{Na}$ Figura 3, verifica-se que durante os primeiros $500 \mathrm{~s}$ de ensaio, a fluidização manteve-se estável em regime explosivo, com a frequência central permanecendo na faixa de 4,0 a $5,0 \mathrm{~Hz}$. A partir desse instante, através de observações visuais realizadas no decorrer do ensaio, verificou-se uma redução do número de bolhas no interior do leito, pois a atomização da suspensão diluída provocou a elevação da umidade no interior do leito, resultando na diminuição gradativa do movimento das partículas até a completa estagnação das mesmas, atingindo assim o regime de leito fixo. Nesta condição de fluidização, o valor da frequência central atinge valores próximos a $6,0 \mathrm{~Hz}$, concordando com outros trabalhos da literatura, tais como PARISE (2008); PARISE et. al. (2009), SILVA et. al. (2011).
Figura 3 - Perfil da frequência central ao longo do processo de secagem de solução diluída de $\mathrm{CaCO}_{3}$. Dados operacionais: $\mathrm{m}_{\text {esferas_alumínio }}=1,6$ $\mathrm{kg}, \mathrm{u}_{\mathrm{o}} / \mathrm{u}_{\mathrm{mf}}=1,4, \mathrm{~T}_{\mathrm{ar}}=70^{\circ} \mathrm{C} ; \mathrm{P}_{\text {atomização }}=0,1 \mathrm{MPa}$, $\mathrm{C}_{\mathrm{CaCO} 3}=9 \%, \mathrm{Q}_{\text {suspensão_CaCO3 }}=12 \mathrm{~mL} / \mathrm{min}$.

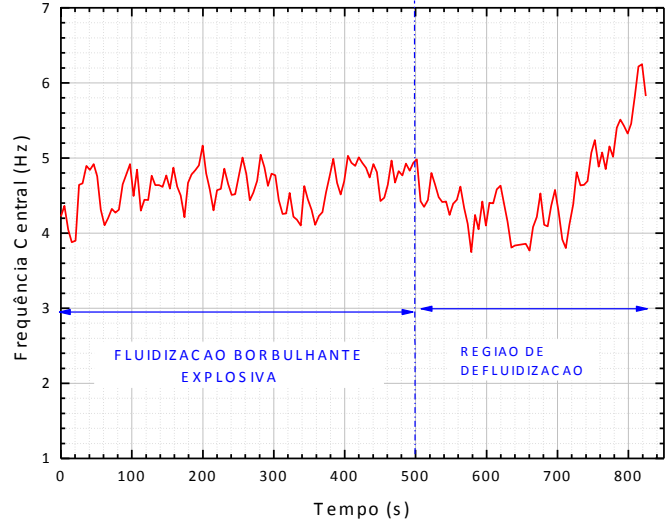

No caso da Figura 4, a fluidização estável foi observada somente por aproximadamente $230 \mathrm{~s}$ de secagem, devido a uma maior vazão de atomização utilizada em relação à da Figura 3. A partir desse momento, observouse uma queda gradual na movimentação das partículas, atingindo o regime de leito fixo após $350 \mathrm{~s}$ de ensaio, onde o valor da frequência central permaneceu na faixa de 5 a $6 \mathrm{~Hz}$.

Figura 4 - Perfil da frequência central ao longo do processo de secagem de solução diluída de $\mathrm{CaCO}_{3}$. Dados operacionais: $m_{\text {esferas alumínio }}=1,6 \mathrm{~kg}$, $\mathrm{u}_{\mathrm{o}} / \mathrm{u}_{\mathrm{mf}}=1,4, \mathrm{~T}_{\mathrm{ar}}=70^{\circ} \mathrm{C} ; \mathrm{P}_{\text {atomização }}=0,1 \mathrm{MPa}$, $\mathrm{C}_{\mathrm{CaCO} 3}=9 \%, \mathrm{Q}_{\text {suspensão_CaCO3 }}=15 \mathrm{~mL} / \mathrm{min}$.

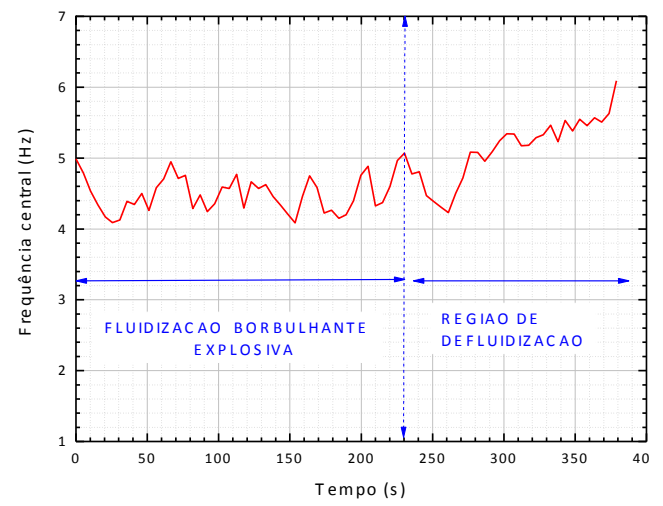

A Figura 5 ilustra a temperatura do leito e das temperaturas obtidas através de balanços 
psicrométricos (bulbo úmido de entrada e saída do ar), e da temperatura de bulbo seco do ar na saída da coluna, referente o ensaio 1 (Tabela 2). A redução no potencial de secagem do leito pode ser verificada em função do abaixamento das temperaturas do leito e do ar de saída e ainda pela aproximação da temperatura de bulbo úmido do ar de saída com o ar de entrada. Do tempo $350 \mathrm{~s}$ ao tempo $550 \mathrm{~s}$, esta aproximação é constante, indicando a secagem da suspensão a taxa constante de evaporação da água. A partir dos $550 \mathrm{~s}$, os sinais de defluidização foram mais pronunciados e a oscilação com tendência de queda da temperatura de bulbo úmido da saída indica também o surgimento de instabilidades no leito.

Figura 5 - Perfil de temperatura do ar e do leito. Dados operacionais: $m_{\text {esferas_alumínio }}=1,6 \mathrm{~kg}$, $\mathrm{u}_{\mathrm{o}} / \mathrm{u}_{\mathrm{mf}}=1,4, \mathrm{~T}_{\mathrm{ar}}=70^{\circ} \mathrm{C} ; \mathrm{P}_{\text {atomização }}=0,1 \mathrm{MPa}$, $\mathrm{C}_{\mathrm{CaCO} 3}=9 \%, \mathrm{Q}_{\text {suspensão_CaCO3}}=12 \mathrm{~mL} / \mathrm{min}$.

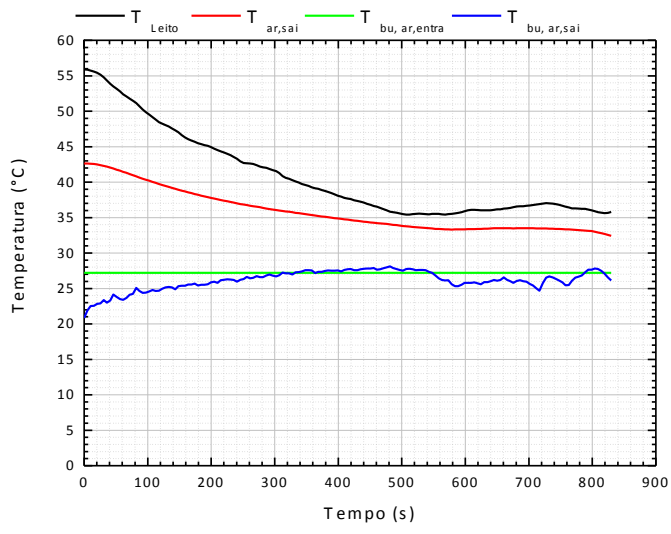

Na Figura 6 são apresentados os perfis das umidades relativa e absoluta na entrada e saída da coluna de fluidização. Verifica-se que, em torno de $500 \mathrm{~s}$ de experimento, as umidades relativa do ar e absoluta na saída da coluna de fluidização alcançam valores máximos de $64 \%$ e $0,026 \mathrm{~kg}$ de água $/ \mathrm{kg}$ de ar seco, respectivamente. A partir desse instante, o leito encontra-se na região de defluidização e observa-se uma oscilação pronunciada dessas umidades, que pode ser ocasionada pela redução da movimentação das partículas.
Figura 6 - Perfil de umidade do ar. Dados operacionais: $\mathrm{m}_{\text {esferas_alumínio }}=1,6 \mathrm{~kg}, \mathrm{u}_{\mathrm{o}} / \mathrm{u}_{\mathrm{mf}}=1,4$, $\mathrm{T}_{\mathrm{ar}}=70^{\circ} \mathrm{C} ; \mathrm{P}_{\text {atomização }}=0,1 \mathrm{MPa}, \mathrm{C}_{\mathrm{CaCO} 3}=9 \%$, $\mathrm{Q}_{\text {suspensão_CaCO3 }}=12 \mathrm{~mL} / \mathrm{min}$.

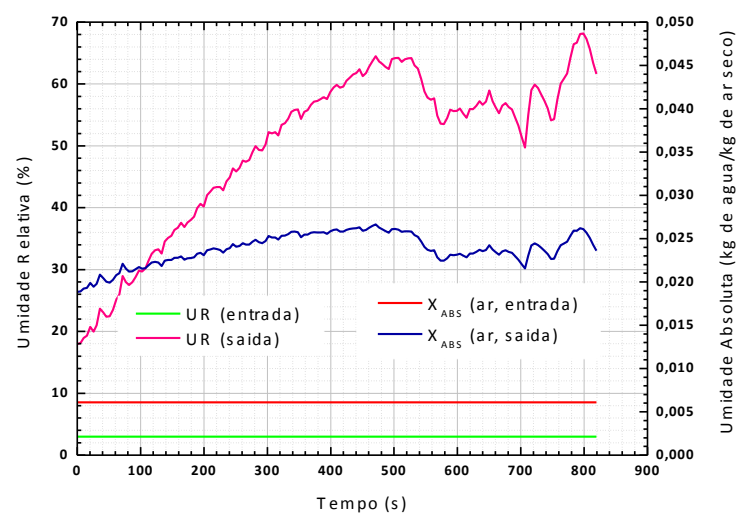

\section{CONCLUSÕES}

Neste trabalho foi realizado um estudo da fluidodinâmica do leito fluidizado durante o processo de secagem de suspensão diluída de carbonato de cálcio, através da análise espectral Gaussiana. Partindo o processo em regime de fluidização do tipo borbulhante explosiva, onde os valores de frequencia central permaneceram na faixa de 4,0 e $5,0 \mathrm{~Hz}$, foi possível verificar a alteração do estado fluidodinâmico na região de defluidização utilizando a análise espectral Gaussiana, atingindo o leito fixo, com frequencia central em torno de 6,0 $\mathrm{Hz}$. Adicionalmente, pode-se observar a redução potencial de secagem pelo decréscimo das temperaturas do leito e do ar de saída e através da tendência de aproximação da temperatura de bulbo úmido do ar de saída com o ar de entrada. Verificou-se que na região de fluidização estável, as umidades relativa do ar e absoluta na saída da coluna alcançaram valores máximos de $64 \%$ e 0,026 $\mathrm{kg}$ de água/kg de ar seco, respectivamente.

\section{NOMENCLATURA}


A Amplitude de modulação

$A_{e}\left(f_{k}\right)$ Amplitude espectral medida $(\mathrm{Pa})$

$\mathrm{C}$ Concentração (massa de sólido/volume de suspensão)

$\mathrm{d}_{\mathrm{p}} \quad$ Diâmetro médio das partículas ( $\mathrm{mm}$ )

$\mathrm{e}_{\mathrm{k}} \quad$ Resíduo da curva de ajuste

$\mathrm{f}_{\mathrm{k}} \quad$ Frequência do sinal $(\mathrm{Hz})$

$\mathrm{f}_{\mathrm{m}} \quad$ Frequência central $(\mathrm{Hz})$

$\mathrm{G}\left(\mathrm{f}_{\mathrm{k}}\right)$ Curva de distribuição normal

$\mathrm{m} \quad$ Massa $(\mathrm{kg})$

$\mathrm{P}_{\text {atomização }}$ Pressão de atomização $(\mathrm{Pa})$

Q Vazão volumétrica ( $\mathrm{mL} / \mathrm{min}$ )

$\mathrm{u}_{\mathrm{o}} \quad$ Velocidade superficial do gás $(\mathrm{m} / \mathrm{s})$

$\mathrm{u}_{\mathrm{mf}}$ Velocidade de mínima fluidização $(\mathrm{m} / \mathrm{s})$

$\mathrm{T}_{\mathrm{ar}} \quad$ Temperatura do ar na entrada do leito $\left({ }^{\circ} \mathrm{C}\right)$

$\mathrm{T}_{\text {ar,sai }}$ Temperatura do ar na saída da coluna $\left({ }^{\circ} \mathrm{C}\right)$

$\mathrm{T}_{\text {bu }} \quad$ Temperatura de bulbo úmido $\left({ }^{\circ} \mathrm{C}\right)$

$\mathrm{T}_{\text {leito }} \quad$ Temperatura do leito $\left({ }^{\circ} \mathrm{C}\right)$

UR Umidade relativa do ar (\%)

$\mathrm{X}_{\mathrm{abs}} \quad$ Umidade absoluta ( $\left.\mathrm{kg}_{\text {água }} / \mathrm{kg}_{\text {ar seco }}\right)$

$\sigma$ Desvio padrão da distribuição espectral $(\mathrm{Hz})$

\section{REFERÊNCIAS}

BAI, B., et al. Characterization of the void size distribution in fluidized beds using statistics of pressure fluctuations. Powder Technology, v.160, p. $81-92,2005$

BOOK, G. et al. On-line detection of bed fluidity in gas-solid fluidized beds with liquid injection by passive acoustic and vibrometric methods. Powder Technology, v. 205, p.126136, 2011.

BRIENS, C. et al. On-line detection of bed fluidity in a fluidized bed coker. Powder Technology, v. 138, p. 160-168, 2003

\section{MARONGA, S. On the Optimization of the} Fluidized Bed Particulate Coating
Process. 1998. 78 f. Ph.D.Thesis - Course of Chemical Engineering, Department Of Chemical Engineering And Technology, Royal Institute Of Technology, Stockholm, 1998.

PARISE, M. R. Aplicação Da Distribuição Espectral Normal Em Leito Fluidizado Gás-Sólido. 2007. 131 f. Tese (Doutorado) Curso de Engenharia Química, Departamento de Faculdade de Engenharia Química, Universidade Estadual de Campinas, Campinas, 2007.

PARISE, M. R. et al. Detection of the minimum gas velocity region using Gaussian spectral pressure distribution in a gas-solid fluidized bed. Powder Technology, Campinas, v. 182, n. 3, p.453458, 10 mar. 2008.

SHABANIAN, J. CHAOUKI, J. Hydrodynamics of a gas-solid fluidized bed with thermally induced interparticle forces, Chemical Engineering Journal, v. 259, p.135-152, 2015.

SILVA, C.A.M. et al. Monitoramento do fenômeno da defluidização no processo de recobrimento em leito fluidizado gás-sólido. In: XXXIV CONGRESSO BRASILEIRO DE SISTEMAS PARTICULADOS. 2009. Campinas. Anais...Campinas: SP, 2009.

SILVA, C. A. M. et al. Control of fluidized bed coating particles using Gaussian spectral pressure distribution. Powder Technology, Campinas, v. 212, p.445-458, 25 out. 2011.

SILVA, C. A. M. et al. Monitoring and control of coating and granulation processes in fluidized beds - A review. Advanced Powder Technology, v. 25, p.195-210, 2014. 
SILVA, C.A.M. Aplicação de tecnologias analíticas de processo e inteligência artificial para monitoramento e controle de processo de recobrimento de partículas em leito fluidizado. 2015. $301 \mathrm{f}$. Tese (Doutorado) - Curso de Engenharia Química, Departamento de Faculdade de Engenharia Química, Universidade Estadual de Campinas, Campinas, 2015.

\section{AGRADECIMENTOS}

Os autores agradecem o Conselho Nacional de Desenvolvimento Científico e Tecnológico (CNPq), a Fundação Araucária e Universidade Tecnológica Federal do Paraná Campus Ponta Grossa pelo suporte financeiro para a realização deste trabalho. 